\title{
Preisträger und Ehrenmitglieder der DRG 2019
}

\section{Ehrenmitgliedschaften der Deutschen Röntgengesell- schaft 2019}

\section{Prof. Dr. Kassa Darge}

(Philadelphia, USA)

\section{o. Univ.-Prof. Dr. Christian Herold (Wien, Österreich)}

\section{o. Univ.-Prof. Dr. Werner Jaschke (Innsbruck, Österreich)}

\section{Prof. Dr. Zhengyu Jin}

(Peking, China)

\section{Preisträger der DRG 2019}

\section{Alfred-Breit-Preis}

Der Preis erinnert seit 2012 an Prof. Alfred Breit, einen der Wegbereiter der Strahlentherapie. Der Preis wird vergeben für Arbeiten in der radiologischen Forschung, die zu Fortschritten in der Krebstherapie beigetragen haben.

Prof. Dr. Katrine Riklund (Umeå, Schweden) für ihre herausragenden wissenschaftlichen Arbeiten auf dem Gebiet der Radiologie.

\section{Wachsmann-Innovations-Preis}

Der Wachsmann-Innovations-Preis wird in Anerkennung der engagierten Weiterentwicklung und erfolgreichen Implementierung von zentralen Angeboten, Leistungen und Programmen der Deutschen Röntgengesellschaft verliehen. In diesem Jahr wird mit dem Preis das Team der Deutschen Gesellschaft für Interventionelle Radiologie und minimalinvasive Therapie (DeGIR) und der Deutschen Gesellschaft für Neuroradiologie (DGNR) gewürdigt, das mit dem Aufbau und der Weiterentwicklung eines Zertifizierungssystems in der interventionellen Radiologie einen Standard gesetzt hat, der zunehmend von den Akteuren im Gesundheitssystem anerkannt wird.

Der Wachsmann-Innovations-Preis ist mit $500 €$ dotiert.

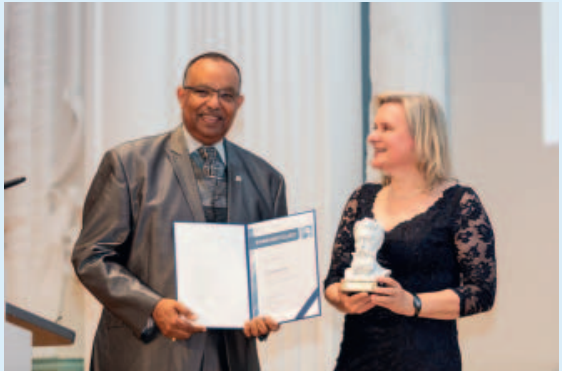

Ehrenmitglied Prof. Dr. Kassa Darge (li.) und Kongresspräsidentin Prof. Dr. Gundula Staatz. @ D DRG/Jörg Singer

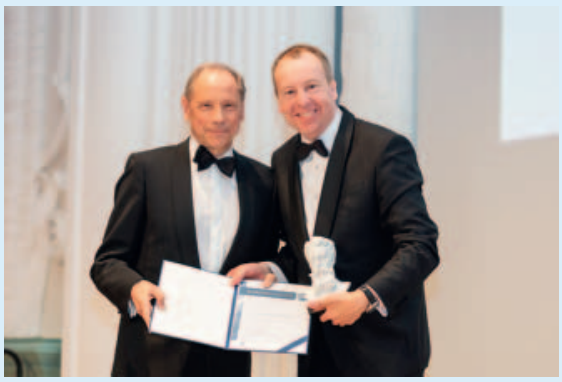

Ehrenmitglied o. Univ.-Prof. Dr. Christian Herold (li.) und DRG-Vizepräsident Prof. Dr. Stefan O. Schönberg. @ DRG/Jörg Singer

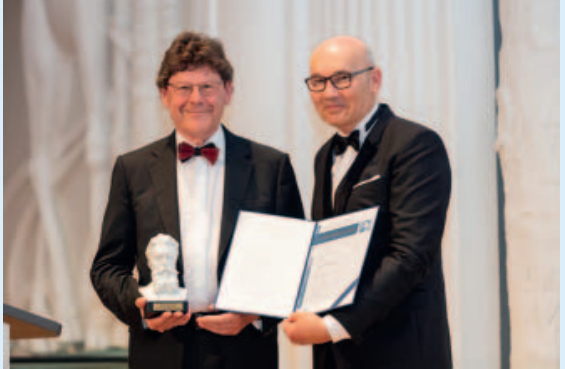

Ehrenmitglied o. Univ.-Prof. Dr. Werner Jaschke (li.) und Kongresspräsident Prof. Dr. Walter Heindel. @ DRG/Jörg Singer

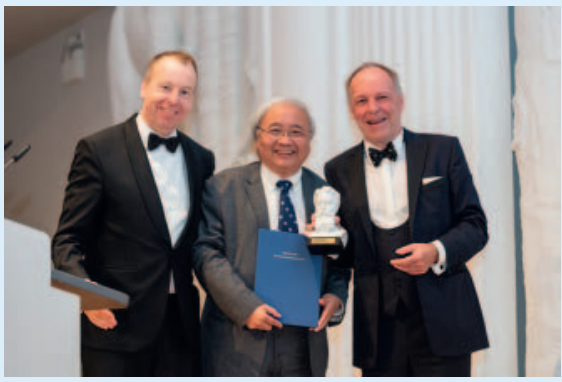

Ehrenmitglied Prof. Dr. Zhengyu Jin (Mitte) mit DRG-Vizepräsident Prof. Dr. Stefan O. Schönberg (li.) und Kongresspräsident Prof. Dr. Michael Forsting. @ D DRG/Jörg Singer

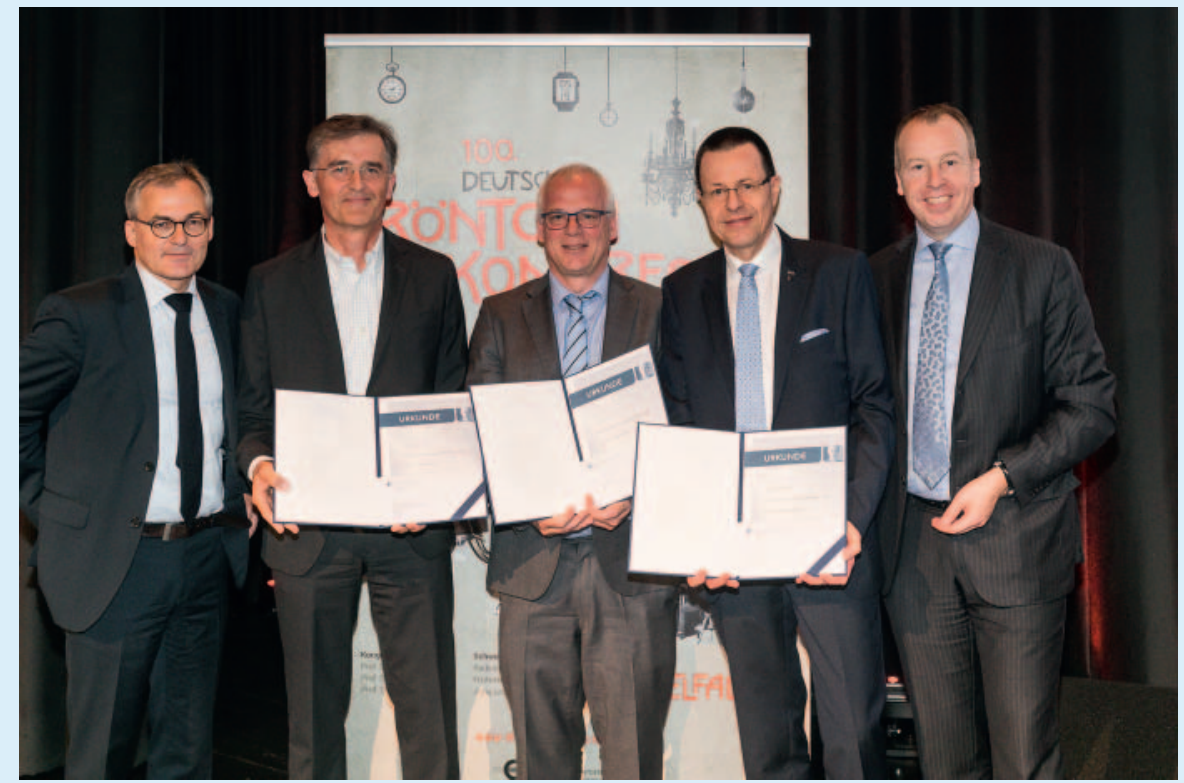

Die Laudatoren Prof. Dr. Arnd Dörfler (li.) und Prof. Dr. Stefan O. Schönberg (re.) verliehen die Wachsmann-Innovations-Preise an (Mitte, v. I. n. r.): Prof. Dr. Peter Reimer, Prof. Dr. Ansgar Berlis und Prof. Dr. Peter Landwehr sowie an Prof. Dr. Andreas Mahnken und Prof. Dr. Werner Weber, die beide an der Verleihung nicht teilnehmen konnten. @ DRG/Thomas Rafalzyk 


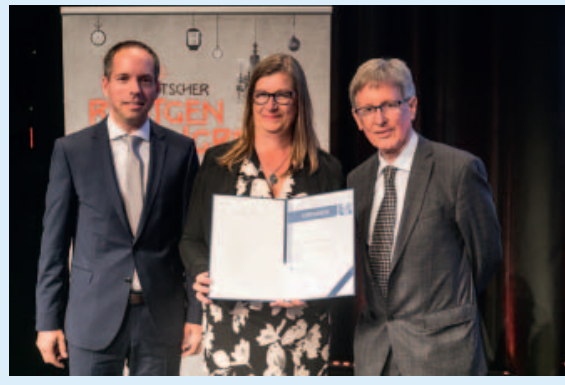

Prof. Dr. Katrine Riklund (Mitte) mit DRGPräsident Prof. Dr. Gerald Antoch (li.) und Laudator Prof. em. Dr. Dr. h.c. Michael Molls (re.). @DRG/Thomas Rafalzyk

Prof. Dr. Ansgar Berlis (Augsburg)

Prof. Dr. Peter Landwehr (Hannover)

Prof. Dr. Andreas Mahnken (Marburg)

Prof. Dr. Peter Reimer (Karlsruhe)

Prof. Dr. Werner Weber (Bochum)

\section{Marie-Curie-Ring}

Der Marie-Curie-Ring zeichnet eine/n herausragende/n junge/n Wissenschaftler/in der Radiologie aus. Grundlage der Begutachtung sind die gesamten bisherigen Leistungen, die im besonderen Maße befähigen sollen, eine herausragende wissenschaftliche Laufbahn zu vollenden.

PD Dr. Peter Bannas (Hamburg) in Anerkennung seiner herausragenden Leistungen auf dem Gebiet der Radiologie.

\section{Wilhelm-Conrad-Röntgen-Preis}

Zum Gedenken an ihren Namensgeber vergibt die Deutsche Röntgengesellschaft seit 1979 jährlich den Wilhelm-Conrad-Röntgen-Preis.

Dr. Dr. Michel Eisenblätter (Freiburg) für seine wissenschaftliche Arbeit „Bildgebung von Immunzellmigration und -aktivität in Entzündung und Tumorerkrankung“.

\section{Röntgen-Vorleser}

Sascha Lobo (Berlin): „Radiologie \& digitaler Wandel“"

\section{Werner-Porstmann-Preis}

In Gedenken an Werner Porstmann (1921 1982), einen Pionier der kardiovaskulären Interventionen, schreiben die Deutsche Röntgengesellschaft und die Deutsche Gesellschaft für Interventionelle Radiologie
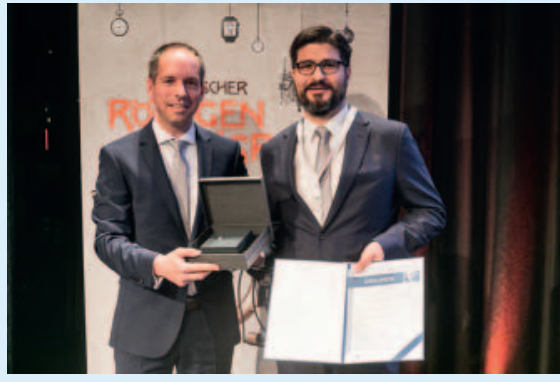

Laudator Prof. Dr. Gerald Antoch (li.) bei der Verleihung des Marie-Curie-Rings an PD Dr. Peter Bannas (re.). @DRG/Thomas Rafalzyk

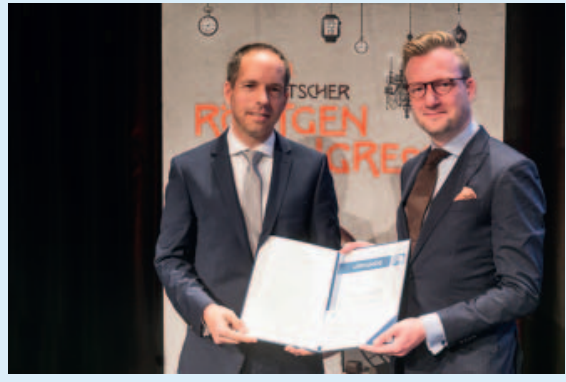

Laudator Prof. Dr. Gerald Antoch (li.) verleiht Dr. Dr. Michel Eisenblätter den Wilhelm-Conrad-Röntgen-Preis. CDRG/Thomas Rafalzyk

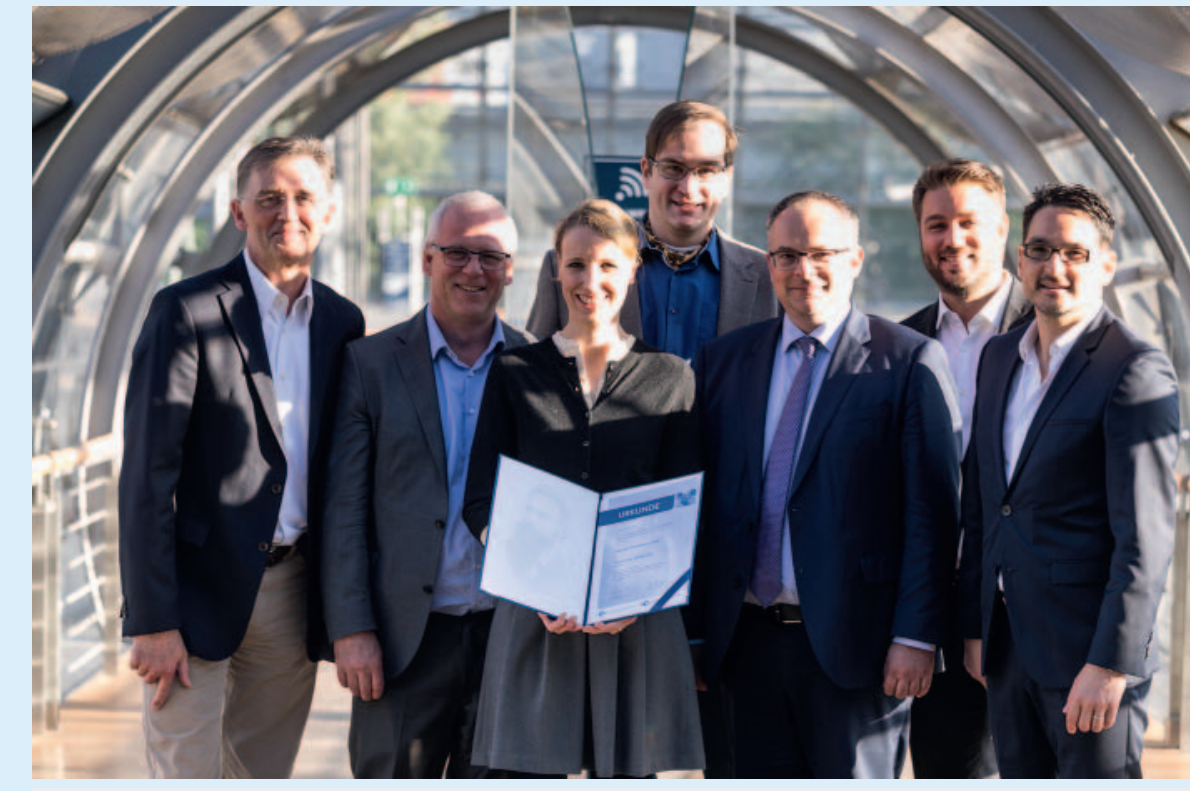

Preisträgerin PD Dr. Wibke Uller (Mitte) mit dem DeGIR-Vorstand. CDRG/Thomas Rafalzyk und minimalinvasive Therapie (DeGIR) den Werner-Porstmann-Preis zur Förderung der Interventionellen Radiologie aus.

PD Dr. Wibke Uller (Regensburg) für ihre wissenschaftliche Arbeit „Ethanolgel sclerotherapy of venous malformations improves health-related quality-of-life in adults and children - results of a prospective study“.

\section{Walter-Friedrich-Preis}

Der Walter-Friedrich-Preis berücksichtigt insbesondere die Gemeinsamkeit von Physik, Technik, Biologie und medizinischer Radiologie.

Dr. Philipp M. Kazmierczak (München) für die wissenschaftliche Arbeit „Untersuchung funktioneller und molekularer Imaging-Bio- marker zum Monitoring zielgerichteter Tumortherapien im Tiermodell“.

\section{Promotionspreis}

Der Promotionspreis der Deutschen Röntgengesellschaft wurde erstmals zum Jahr 2012 ausgeschrieben, um wissenschaftlich engagierte Studierende und Mediziner/innen zu motivieren und zu fördern. Im Fall einer Auszeichnung der eingereichten Arbeit wird diese als Originalarbeit in der Fachzeitschrift RöFo veröffentlicht.

Dr. Martin Sinn (Hamburg) für seine wissenschaftliche Arbeit „Diagnostische Genauigkeit der Differenzierung zwischen akutem und chronischem Myokardinfarkt durch T1- und T2-Maps“. 


\section{Young-Investigator-Award}

Dr. Corinna Storz (Tübingen) für ihre wissenschaftliche Arbeit „Vergleich von T1Mapping und myokardialer Strain-Analyse in der CMRT: Zusammenhang des extrazellulären Volumens mit myokardialem Strain in einer gesunden Bevölkerungsstichprobe“.

\section{Dr. Katerina Deike-Hofmann (Heidelberg)} für ihre wissenschaftliche Arbeit "Gadolinium-haltige Kontrastmittel im glymphatischen System: Ignoriert und fehlinterpretiert".

\section{Bracco-Posterpreis}

PD Dr. Christian Fischer (Heidelberg) wird für seine wissenschaftlichen Posterpräsentationen auf dem Gebiet des kontrastverstärkten Ultraschalls mit 2 Bracco-Posterpreisen ausgezeichnet.

\section{Postertitel:}

„Kontrastmittelverstärkter Ultraschall (CEUS) zur Beurteilung der tibialen Knochenperfusion bei physiologischer und gestörter Frakturheilung mit Pseudarthrosenbildung"

„Kontrastmittelverstärkter Ultraschall (CEUS) zur Muskelbeurteilung nach Supraspinatussehnennaht"

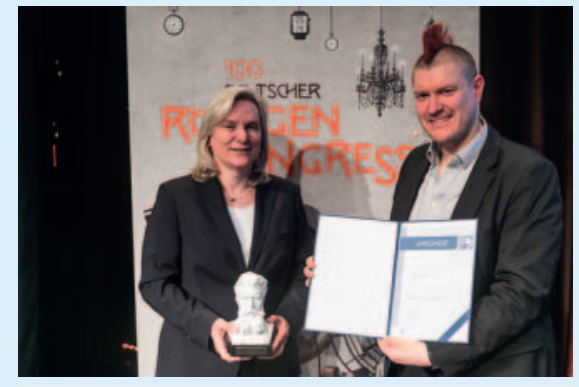

Kongresspräsidentin Prof. Dr. Gundula Staatz (li.) mit Röntgen-Vorleser Sascha Lobo. @DRG/Thomas Rafalzyk

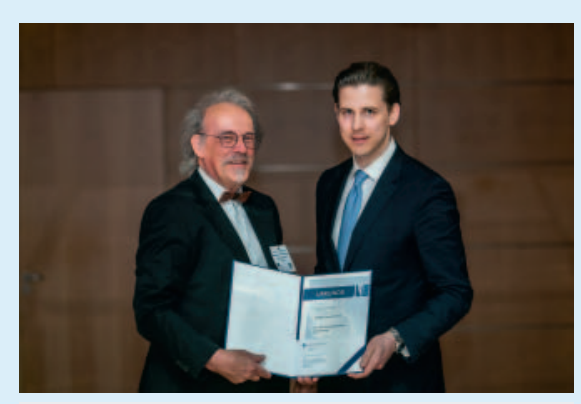

Laudator Dr. Michael Wucherer mit Preisträger Dr. Philipp Kazmierczak. CDRG/Sera Zöhre Kurc

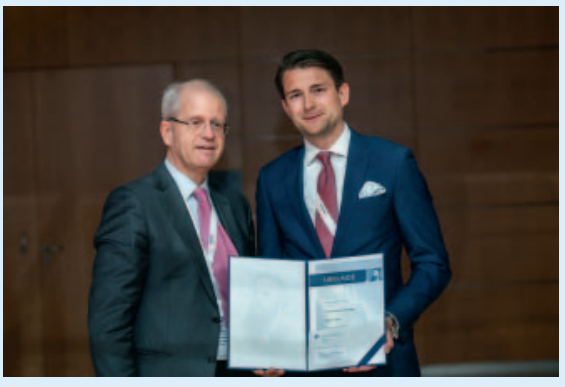

Dr. Martin Sinn (re.) mit Laudator Prof. Dr. Gerhard Adam. @ DRG/Sera Zöhre Kurc

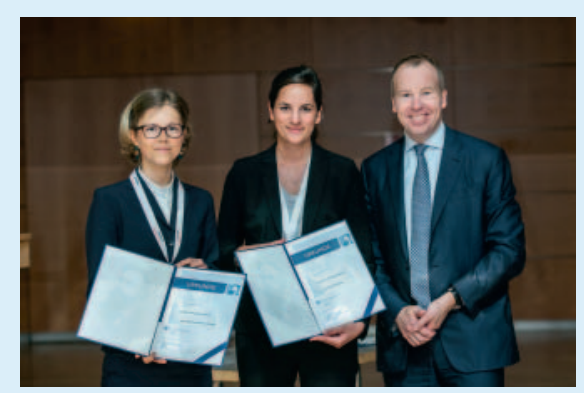

Die Preisträgerinnen des Young-Investigator-Awards, Dr. Katerina Deike-Hofmann (li.) und Dr. Corinna Storz (Mitte), mit Laudator Prof. Dr. Stefan O. Schönberg. ODRG/Sera Zöhre Kurc 